\title{
SCIDiC
}

\author{
International Journal of Dentistry and Oral Science (IJDOS) \\ ISSN: 2377-8075
}

\section{Evaluation of Root Canal Morphology of Lower Lateral Incisors using Cone Beam Computed Tomography in an Indian Subpopulation}

Research Article

Jerry Jose ${ }^{1}$, Ajitha $\mathrm{P}^{2 *}$

${ }^{1}$ Post Graduate Student, Department of Conservative Dentistry and Endodontics, Saveetha Dental College, Saveetha Institute of Medical and Technical Sciences, Saveetha University, Chennai, India.

${ }^{2}$ Professor, Department of Conservative Dentistry and Endodontics, Saveetha Dental College, Saveetha Institute of Medical and Technical Sciences, Saveetha University, Chennai, India.

\section{Abstract}

\begin{abstract}
Aim: To aim of the current study was to evaluate the root canal morphology of lower lateral incisors in an Indian subpopulation using Cone-Beam computed tomography.

Material and Methods: The CBCT images of 256 patients were obtained from the Department of Oral Medicine and Radiology of the University. Various parameters such as number of roots, number of root canal and canal configuration were evaluated and recorded and then classified according to Vertucci's classification of root canals.

Results: The most common root canal morphology is for Vertucci's Type 1 which is $40.2 \%$, and Type 2 being $33.6 \%$. The other types being Type 3(11.4\%), Type 4(6.3\%), Type 5(1.9\%), Type 7(4.7\%) and Type 8(1.9\%).

Conclusion: From the assessed data it was seen the root canal morphology of lower lateral incisors exhibited a higher incidence of Vertucci's Type 1 and Type 2 andother configurations being Type 3, Type 4, Type 7, Type 5, Type 8. Type 6 was not to be seen in the assessed sample. The variation in the root canal morphology was seen most commonly in the male population than in female population.
\end{abstract}

Keywords: Lower Lateral Incisors; Root Canal Morphology; Cone Beam Computed Tomography; Vertucci’s Classification.

\section{Introduction}

Endodontic treatment is a complex process which requires the practitioners to study the root canal morphology in tremendous detail. Accurate diagnosis of the root canal morphology is of very high significance since it can lead to a successful endodontic treatment [1]. Root canal therapy involves shaping and cleaning of root canal systemfollowed by filling with an inert restorative material. Presence of untreated canal is said to be a major cause of endodontic failure [2]. Different radiographic methods are used for endodontic diagnosis of root canal morphology such as tooth staining and colouring, conventional radiographs, digital medium advanced radiographic techniques and the more recent computed tomography techniques [3].
Cone beam computed tomography or CBCT is amethod introducedfor diagnostic application in endodontic clinical practice. It is used for different applications such as assessment of endodontic trauma, diagnosis of radiographic signs of periapical lesions, confirmation of nonodontogenic causes of pathosis, assessment of complex root canal systems prior to endodontic management, assessment of endodontic treatment complications or for management of root resorption [4]. CBCT is said to be used initially for angiography to assess the blood patterns and has paved its way into various medical applications in different fields. Its mechanism is said to be acquire individual image slices which are reconstructed and made into a single image. CBCT is said to obtain a single field of view (FOV) in an arc manner of planar image projection [5]. The smaller the FOV the less the radiation exposure seen to the patient. Another advantage of CBCT over conventional CT is the decreased scan time ranging from $10-40$ secs and in current

\author{
*Corresponding Author: \\ Ajitha $P$, \\ Professor, Department of Conservative Dentistry and Endodontics, Saveetha Dental College, Saveetha Institute of Medical and Technical Sciences, Saveetha University, Chennai, \\ India. \\ Tel: +919444174551 \\ E-mail: ajitharijesh@gmail.com \\ Received: Ocotber 08,2020 \\ Accepted: December 02, 2020 \\ Published: December 10, 2020 \\ Citation: Jerry Jose, Ajitha P. Evaluation of Root Canal Morphology of Lower Lateral Incisors using Cone Beam Computed Tomography in an Indian Subpopulation. Int J Dentistry
} Oral Sci. 2020;7(12):1193-1196. doi: http://dx.doi.org/10.19070/2377-8075-20000236

Copyright: Ajitha $\mathbf{P}^{\circ}$ 2020. This is an open-access article distributed under the terms of the Creative Commons Attribution License, which permits unrestricted use, distribution and reproduction in any medium, provided the original author and source are credited. 
advanced systems ranging from $5-8$ seconds [6].

Various retrospective studies [7-9] have been done which aims at evaluating the different root canal morphology in different subpopulation but none of the studies have been done to evaluate the root canal variations of lower lateral incisors using Cone-beam computed tomography in an Indian subpopulation. The current study aims to evaluate the root canal morphology of lower lateral incisors in an Indian subpopulation (Chennai) using cone beam computed tomography data.

\section{Materials and Methods}

The CBCT images were obtainedfrom Dentsply Sirona 3D unit (Orthophos XG 3D) with operating parameters(3-6 mA and 60$90 \mathrm{kV}$, Exposure time - 5.1 seconds). The smallest possiblefield of view $(\mathrm{FOV})$ was used $(8 \times 8 \mathrm{~cm})$ and isotropic voxel size was 0.16 mm. The effective dose was between $14 \mu \mathrm{sv}-166 \mu \mathrm{sv}$. All CBCT scans were performed with the minimum exposure necessary for adequate image quality. The as low as reasonably achievable (ALARA) protocol was strictlyfollowed. The patient was made to position parallelto the emitter beam withhorizontal axis towards the alveolar process, and the teethunder examination were placed in thecentreof the volume.Vertucci's classification (1-8) was used as mode of classification to determine the number of canals [10].

A total CBCT data from the time frame April 2019 to September 2019 consisting of 256 patients were collected from the Department of Radiology, out of the 256 patients CBCT data assessed 41 patients CBCT data was excluded. The acquisition process was performed by an experienced radiologist. The data was taken based on the inclusion/exclusion criteria:

\section{Inclusion criteria}

- Permanent mandibular lateral incisors which were fully developed

- Absence of root canal treatment

- Absence of post and core restorations

- Absence of large metallic restorations which may interfere image analysis

- Absence of root resorption or periapical lesions
- No previous root amputation or hemi section

- Presence of high-quality CBCT images in which canal orifice and root canal configuration could be recognized.

\section{Exclusion criteria}

- Distorted CBCT images

- Edentulous ridges

- Patients below the age of 18 years and above the age of 60 years

- Missing lower anterior

- Patients undergoing orthodontic treatment.

The data was collected interpreted digitally using Galaxis Galileo's Viewer 1.9 (Sirona Dental Systems, SICAT GmbH \& Co. KG, USA). The following information was recorded:

- The number of roots

- The root canal configuration the results were acquired, analysed and interpreted according to Vertucci's Classification.

An experienced endodontist was made to assess the CBCT images independently and could scroll through axial, coronal and sagittal views of each tooth. In case of disagreement, the case was made to discuss with another fellow endodontist until a consensus was reached.

\section{Statistical Analysis}

The statistical analysis was done using SPSS 21.0 (IBM Corp, Armonk, USA). Descriptive Analysis was conducted following by which a chi square analysis was conducted on the acquired data among the same patient. A p value less than 0.05 was considered to be statistically significant.

\section{Results}

From the analysed 214 samples, 125 samples belonged to the male population and 90 samples belonged to the female population. All the analysed images showed a single root configuration. The incidence of canal variation was seen to be around $60 \%$ from the total assessed samples. Table 1 denotes the frequency of canal variation from the assessed samples. Vertucci's Type 1 was seen

Table 1. Frequency of distribution seen in the assessed CBCT images.

\begin{tabular}{|c|c|c|c|c|c|c|c|}
\hline & & & \multicolumn{2}{|c|}{ Tooth Number } & \multirow{2}{*}{ Total } & \multirow{2}{*}{ Chi square } & \multirow{2}{*}{$\mathrm{p}$ value } \\
\hline & & & 32 & 42 & & & \\
\hline \multirow{14}{*}{ Observer } & \multirow{2}{*}{ Type 1} & $\mathrm{~N}$ & 76 & 96 & 172 & \multirow{16}{*}{20.16} & \multirow{16}{*}{$0.003^{*}$} \\
\hline & & $\%$ & $35.50 \%$ & $44.90 \%$ & $40.20 \%$ & & \\
\hline & \multirow{2}{*}{ Type 2} & $\mathrm{~N}$ & 87 & 57 & 144 & & \\
\hline & & $\%$ & $40.70 \%$ & $26.60 \%$ & $33.60 \%$ & & \\
\hline & \multirow{2}{*}{ Type 3} & $\mathrm{~N}$ & 19 & 30 & 49 & & \\
\hline & & $\%$ & $8.90 \%$ & $14.00 \%$ & $11.40 \%$ & & \\
\hline & \multirow{2}{*}{ Type 4} & $\mathrm{~N}$ & 17 & 10 & 27 & & \\
\hline & & $\%$ & $7.90 \%$ & $4.70 \%$ & $6.30 \%$ & & \\
\hline & \multirow{2}{*}{ Type 5} & $\mathrm{~N}$ & 1 & 7 & 8 & & \\
\hline & & $\%$ & $0.50 \%$ & $3.30 \%$ & $1.90 \%$ & & \\
\hline & \multirow{2}{*}{ Type 7} & $\mathrm{~N}$ & 8 & 12 & 20 & & \\
\hline & & $\%$ & $3.70 \%$ & $5.60 \%$ & $4.70 \%$ & & \\
\hline & \multirow{2}{*}{ Type 8} & $\mathrm{~N}$ & 6 & 2 & 8 & & \\
\hline & & $\%$ & $2.80 \%$ & $0.90 \%$ & $1.90 \%$ & & \\
\hline \multirow{2}{*}{\multicolumn{2}{|c|}{ Total }} & $\mathrm{N}$ & 214 & 214 & 428 & & \\
\hline & & $\%$ & $100.00 \%$ & $100.00 \%$ & $100.00 \%$ & & \\
\hline
\end{tabular}


to $35.5 \%$ in tooth 32 and $44.9 \%$ in tooth 42 , Vertucci's Type 2 was seen to be second highest in $32(40.7 \%)$ and $42(26.6 \%)$. A total variation canal variation was seen of $33.6 \%$. Vertucci's Type 3 was seen to be the next most commonly seen with $32(8.9 \%)$ and $42(14.0 \%)$. Vertucci's Type 4 was seen to be $6.3 \%$, Vertucci's Type 5 was seen to be $1.9 \%$, Vertucci's Type 7 was seen to $4.7 \%$ and Vertucci's Type 8 was seen to $1.9 \%$ respectively. A Chi-square test between the analysed samples showed a significant correlation between the assessed samples $(\mathrm{p}<0.05)$.

\section{Discussion}

The current study aims to assess the diverse root canal morphology in an Indian subpopulation. Mandibular lateral incisors are one of the most common teeth present in the oral cavity shown to have a high degree of root canal morphology variations and has proven to clinically challenging for various practitioners. The incidence of second canal is seen more than $40 \%$ with more than $1 \%$ having a separate foramen [5]. Numerous studies have shown the presence of varied root canal morphology for lower anterior $[11,12]$. It is seen that root canal morphology variation is seen among different racial population as well with the results varying among different population. This could possibly be due to genetic diversity playing a role in the variation of the canals. CBCT is an effective tool which can be used for the endodontic practice for locating additional canals or varied tooth morphology. Different scenarios are seen in which the mandibular lateral incisor exhibit varied root canal morphology. CBCT imaging is a non-invasive technique that was reported to be as accurate as the modified canal staining and clearing technique for evaluating root canal systems [13]. Root canal variation is one of the factors seen with studies showing the incidence of anatomical variation being more common in males than in females [14]. The current study also corelates with the existing literature of the canal variation being more diverse in male population than in female population.

Several studies have used other different methods for the examination of root canal morphology which are use of polyester resins, creating transparent angles and use of 2D radiographs [15]. In clinical practice the use of roentogram is said to have severe limitations due to fact that it is 2-dimensional in nature which limits the clinician's ability to detect any additional canals. Currently with the introduction of CBCT the clinician can visualize the tooth in a 3-dimensional view which helps them to acquire the image. In contrast to traditional radiography, CBCT scanning provides 3-dimensional images in axial, sagittal, and coronal sections that could avoid geometric distortion and anatomic super imposition. However, the cost of CBCT scanning compared with periapical radiography hinder its clinical application in developing countries. A case report published by Reeh et al, has seen the incidence of fusion of the mandibular lateral incisor and seen varied apices with some being more than one [16]. One of the main failures of endodontic treatment is the missed root canal system this is seen due to the anatomical variation in the root canal system [17]. A study done by Boruah et al., [18] in which the incidence of type 1 root canal morphology is $63.5 \%$, another study done by Wu et al., [19] for the Taiwanese population had seen similar results with Vertucci's type 1 being 75\% and type 3 being 23\%. The present study shows a slight variation in the results obtained in which Vertucci's Type 1 (40.2\%), Type 2 (33.6\%), Type 3(11.4\%), Type $4(6.3 \%)$, Type $5(1.9 \%)$, Type $7(4.7 \%)$ and Type $8(1.9 \%)$. The in- cidence of a type 6 canal configuration was seen to nil from the assessed samples.

Previously, various methods were advocated for the examination of root canal morphology. The most primitive method used were to sectionthe tooth, this was discontinued due to fact that it does not allow a continuous view of the root canal system and also irreversibly changing the morphology [20]. Another method given by Robertson et al.which was used for a short amount of time is the clearing technique in which India ink was used as for the identification of the root canal system [21]. Ground sectioning of the apices has also been done by various authors to study the root canal morphology. In the recent timesscanning electron microscopy or SEM is another method used to for the study of root canal morphology but clinically impossible have not been able to use [22]. With the advent of cone beam computed tomography, the clinician can evaluate and plan the data with considerable detail the mechanism of CBCT being a fan shaped beam which is exposed to the target structure which is simulated to multiple exposure in a single field of view and involving the sectioning of the tooth in three planar directionthat is axial, coronal and sagittal direction [23]. The mechanism of CBCT radiographs is very much similar to conventional OPG but unlike the conventional orthopanthograph which is taken in a 2-dimensional image CBCT acquires the image in 3-dimensional in which a gantry is used to acquire slices of images which are compiled together digitally to produce a single image. Hundreds of planar projection images are acquired in the field of view which ranges at 180 degree [24].

Multiple studies are performed among different ethnic population to determine the root canal morphology. In Iranian population it was seen that the population has Type 1 having $70.6 \%$ for permanent lateral incisors compared to other types [13]. A study done by Altunsoy et al., for the Turkish population had seen that Type 5 was one of the frequently observed root canal configurations [25]. In the Chinese population a study conducted by Liu et al., had seen that males had $14.6 \%$ and females had $11.9 \%$ incidence of second canal [26]. Micro CT is another method which can be used for evaluation of the root canal morphology which is able to provide much accurate information about root canal morphology than the CBCT but its indicative purpose in clinical practice is not applicable, but they can only be used on extracted teeth. In clinical situations conventional radiography is the most commonly used method to distinguish tooth anatomy.

One of the limitations of the present study, is to not use an advanced diagnostic method such as micro CT for the evaluation of root canal morphology. Though micro CT is shown to be much effective in analysing root canal morphology the excessive cost for analysis and its clinical application is seen to negligible the usage of CBCT is still preferred for cone beam computed tomography analysis.

\section{Conclusion}

Within the limitations of the present study, it can be concluded that most of the tooth examined had Type 1 root canal morphology of mandibular incisors. In tooth exhibiting more than single canal, the Type II canal configuration was the most prevalent followed by Type III, Type IV configuration based on the Vertucci's Classification. The least exhibited root canal morphology being 
additional type 6. Gender variation as also seen in a factor for the variation in root canal morphology with the incidence of second canal being more common in males than in female population.

\section{References}

[1]. Vertucci FJ. Root canal morphology and its relationship to endodontic procedures. Endodontic topics. 2005 Mar; 10(1):3-29.

[2]. Verma P, Love RM. A Micro CT study of the mesiobuccal root canal morphology of the maxillary first molar tooth. Int Endod J. 2011 Mar; 44(3):210-7. PMID: 20880136.

[3]. Neelakantan P, Subbarao C, Subbarao CV. Comparative Evaluation of Modified Canal Staining and Clearing Technique, Cone-Beam Computed Tomography, Peripheral Quantitative Computed Tomography, Spiral Computed Tomography, and Plain and Contrast Medium-enhanced Digital Radiography in Studying Root Canal Morphology. Journal of Endodontics. 2010 Sep; 36(9): 1547-51. Pubmed PMID: 20728725.

[4]. Patel S, Durack C, Abella F, Roig M, Shemesh H, Lambrechts P, et al. European Society of Endodontology position statement: The use of CBCT in Endodontics. International Endodontic Journal. 2014 Jun; 47(6): 502-4. Pubmed PMID: 24815882.

[5]. Scarfe WC, Farman AG. What is Cone-Beam CT and How Does it Work? Dental Clinics of North America. 2008 Oct;52(4):707-30. Pubmed PMID: 18805225.

[6]. Patel S. New dimensions in endodontic imaging: Part 2. Cone beam computed tomography. International Endodontic Journal. 2009 Jun; 42(6): 463-75. Pubmed PMID: 19298576.

[7]. Lin Z, Hu Q, Wang T, Ge J, Liu S, Zhu M, et al. Use of CBCT to investigate the root canal morphology of mandibular incisors. Surg Radiol Anat. 2014 Nov;36(9):877-82. Pubmed PMID: 24515289.

[8]. Han T, Ma Y, Yang L, Chen X, Zhang X, Wang Y. A Study of the Root Canal Morphology of Mandibular Anterior Teeth Using Cone-beam Computed Tomography in a Chinese Subpopulation. Journal of Endodontics. 2014 Sep;40(9):1309-14. Pubmed PMID: 25043332.

[9]. Saati S, Shokri A, Foroozandeh M, Poorolajal J, Mosleh N, Saati S, et al. Root Morphology and Number of Canals in Mandibular Central and Lateral Incisors Using Cone Beam Computed Tomography. Brazilian Dental Journal. 2018 May;29(3):239-44. Pubmed PMID: 29972448.

[10]. Vertucci FJ. Root canal anatomy of the human permanent teeth. Oral Surgery, Oral Medicine, Oral Pathology. 1984 Nov;58(5):589-99. Pubmed PMID: 6595621.

[11]. Kabak YS, Abbott PV. Endodontic treatment of mandibular incisors with two root canals: Report of two cases. Australian Endodontic Journal. 2007 Apr;33(1):27-31. Pubmed PMID: 17461838.

[12]. Sert $S$, Aslanalp V, Tanalp J. Investigation of the root canal configurations of mandibular permanent teeth in the Turkish population. International Endodontic Journal. 2004 Jul;37(7):494-9. Pubmed PMID: 15189440.

[13]. Aminsobhani M, Sadegh M, Meraji N, Razmi H, Kharazifard MJ. Evalua- tion of the Root and Canal Morphology of Mandibular Permanent Anterior Teeth in an Iranian Population by Cone-Beam Computed Tomography. J Dent (Tehran). 2013 Jul;10(4):358-66. Pubmed PMID: 24396355.

[14]. Mauger MJ, Schindler WG, Walker WA. An evaluation of canal morphology at different levels of root resection in mandibular incisors. Journal of Endodontics. 1998 Sep;24(9):607-9. Pubmed PMID: 9922750.

[15]. Kazemipoor M, Hajighasemi A, Hakimian R. Gender difference and root canal morphology in mandibular premolars: A cone-beam computed tomography study in an Iranian population. Contemp Clin Dent. 2015;6(3):401-4. Pubmed PMID: 26321843.

[16]. Reeh ES, ElDeeb M. Root canal morphology of fused mandibular canine and lateral incisor. Journal of Endodontics. 1989 Jan;15(1):33-5. Pubmed PMID: 2607265.

[17]. Ghamari M, Farhad Mollashahi N, Salarpour M, Mousavi E, Kazemian K, Moudi E, et al. Evaluation of the relationship between crown size and root canal morphology of mandibular incisors by cone beam computed tomography (CBCT). Electron Physician. 2017 Aug 25; 9(8): 5001-7. Pubmed PMID: 28979734.

[18]. Boruah LC, Bhuyan AC. Morphologic characteristics of root canal of mandibular incisors in North-East Indian population: An in vitro study. J Conserv Dent. 2011;14(4):346-50. Pubmed PMID: 22144800.

[19]. Wu Y-C, Cheng W-C, Chung M-P, Su C-C, Weng P-W, Cathy Tsai Y-W, et al. Complicated Root Canal Morphology of Mandibular Lateral Incisors Is Associated with the Presence of Distolingual Root in Mandibular First Molars: A Cone-beam Computed Tomographic Study in a Taiwanese Population. Journal of Endodontics. 2018 Jan;44(1):73-79.e1. Pubmed PMID: 29079050.

[20]. Schneider SW. A comparison of canal preparations in straight and curved root canals. Oral Surgery, Oral Medicine, Oral Pathology. 1971 Aug;32(2):2715. Pubmed PMID: 5284110

[21]. Robertson D, Leeb IJ, McKee M, Brewer E. A clearing technique for the study of root canal systems. Journal of Endodontics. 1980 Jan;6(1):421-4. Pubmed PMID: 7005366.

[22]. Hegde RS, Miller DA. Scanning Electron Microscopy for Studying Root Morphology and Anatomy in Alfalfa Autotoxicity. Agron.j. 1992 Jul;84(4):618-21.

[23]. Mao T, Neelakantan P. Three-dimensional imaging modalities in endodontics. Imaging Sci Dent. 2014 Sep;44(3):177-83. Pubmed PMID: 25279337.

[24]. Scarfe WC, Levin MD, Gane D, Farman AG. Use of cone beam computed tomography in endodontics. Int J Dent. 2009; 2009: 634567. PMID: 20379362.

[25]. Altunsoy M, Ok E, Nur BG, Aglarci OS, Gungor E, Colak M. A cone-beam computed tomography study of the root canal morphology of anterior teeth in a Turkish population. Eur J Dent. 2014;8(3):302-6. Pubmed PMID: 25202207.

[26]. Liu J, Luo J, Dou L, Yang D. CBCT study of root and canal morphology of permanent mandibular incisors in a Chinese population. Acta Odontologica Scandinavica. 2014 Jan 1;72(1):26-30. Pubmed PMID: 24255962. 